\title{
Activation des oncogènes par mutation ponctuelle
}

C'est en changeant les propriétés de son produit que les mutations ponctuelles peuvent activer un oncogène et être à l'origine d'un cancer. Ce mécanisme est probablement en cause dans de nombreux cas de cancérogenèse chimique.

\section{Marie-Henriette}

Loucheux-Lefebvre Directeur de Recherche au Cnrs

Directeur de l'unité Inserm U. 124

- Une mutation ponctuelle est le changement d'une base par une autre.

- Un schéme de la transfection peut être trouvé dans la figure 2 de la réff́rence 1.

*.. Voir lexiques $\mathrm{m} / \mathrm{s} n^{\circ} 2$, vol. 2, p. 104 et $\mathrm{m} / \mathrm{s}$ $n^{\circ} 7$, vol. 2, p. 398 .

\section{ADRESSE}

M.-H. Loucheux-Lefebvre : Inserm U.124 : Expression des gènes et cancérogenèse chimique, Institut de recherches sur le cancer de Lille, 59045 Lille Cedex.

$m / s n^{\circ} 1$ vol. 3, janvier 87 'étude en génétique moléculaire de l'induction et du développement des tumeurs humaines et animales a connu l'essor que l'on sait grâce, d'une part, aux rapides progrès technologiques dont a été l'objet la biologie moléculaire et, d'autre part, à l'élaboration d'une théorie unificatrice du cancer qui reconnaissait le rôle décisif de l'activation de gènes de cancer, ou oncogènes, dans la cancérogenèse, qu'elle soit d'origine virale ou chimique [1]. Deux étapes importantes ont permis la mise en place de cette théorie unificatrice : la première est celle de la découverte des proto-oncogènes cellulaires, qui a mis en évidence le fait que les cellules normales contiennent des gènes très semblables à ceux trouvés dans des virus hautement cancérogènes [2]. La seconde concerne le fait que certains proto-oncogènes peuvent être activés par mutations ponctuelles*, ces dernières ayant été caractérisées sur de l'ADN cellulaire de tumeurs humaines [3-5].

L'activation de ces oncogènes est identifiée grâce aux expériences de transfection qui utilisent la propriété de certaines cellules fibroblastiques d'embryons de souris (NIH 3T3) d'intégrer dans leur génome de l'ADN ajouté au milieu de culture et d'exprimer l'information qu'il code**. Dans la grande majorité des cas, le gène responsable des propriétés transformantes de l'ADN isolé à partir de tumeurs est un protooncogène de la famille ras (Harveyras (H-ras), Kirsten-ras (K-ras), et $\mathrm{N}$-ras). Les quelques oncogènes autres que ras présentant aussi une propriété transformante sont rappelés dans le Tableau I. Il a été montré que l'ADN obtenu à partir d'un certain nombre de tumeurs pouvait dans environ 10 à $20 \%$ des cas [6], induire des modifications morphologiques dans les cultures de cellules NIH 3T3 alors que l'ADN équivalent extrait de tissus normaux ne présentait pas cette propriété.

\section{Activation du gène ras}

Les premiers travaux mettant en évidence l'activation des protooncogènes ayant été réalisés sur des cultures de cellules tumorales, des critiques se sont élevées : elles faisaient remarquer que la propriété transformante aurait pu être acquise à la suite d'un changement secondaire consécutif à l'adaptation, mais ne reflétant pas une propriété de la cellule tumorale de départ $[7,8]$. Depuis, les études réalisées directement sur les tumeurs "fraîches" dont les mutations, après traitement de l'ADN à l'aide des enzymes de restriction, ont été identifiées par hybridation soit avec des sondes clonées soit avec des oligonucléotides***, ont montré qu'une mutation ponctuelle pouvait être 


\section{RÉFÉRENCES}

1. Stéhelin D. Les oncogènes cellulaires, clés de la cancérogenèse. médecine/sciences $1985 ; 1$ : 12-6.

2. Stéhelin D, Varnnus HE, Bishop JM, Vogt PK. DNA related to the transforming gene(s) of avian sarcoma virus is present in normal avian DNA. Nature $1976 ; 260: 170-3$.

3. Reddy EP, Reynolds RK, Santos E, Barbacid $M$. A point mutation is responsible for the acquisition of transforming properties by the T 24 human bladder carcinoma oncogene. Nature 1982 ; 300 : 149-52.

4. Taparowski E, Suard Y, Fasano O, Shimizu K, Goldfarb M, Wigler M. Activation of the $T 24$ bladder carcinoma transforming gene is linked to a single amino acid change. Nature 1982 ; 300 : 762-5.

5. Tabin CJ, Bradley SM, Bargmann CI, et al. Mechanism of activation of a human oncogene. Nature 1982 ; 300 : 143-9.

6. Cooper GM, Lane MA. Cellular transforming genes and oncogenes. Biochim Biophys Acta $1984 ; 738: 9-20$.

7. Duesberg PH. Retroviral transforming gene in normal cells. Nature 1983 ; 304 : 219-26.

8. Rubin H. Mutations and oncogenes cause or effect. Nature $1984 ; 309: 518$

9. Santos E, Martin-Zanca D, Reddy EP, Pierotti MA, Della Porta G, Barbacid M. Malignant activation of a $\mathrm{K}$-res oncogene in lung carcinoma but not in normal tissue of the same patient. Science 1984 ; 223 : 661-4.

10. Fujita J, Yoshida O, Yuasa Y, Rhim JS, Hatanaka M, Aaronson S. Ha-ras oncogenes are activated by somatic alterations in human urinary tract tumours. Nature 1984; 309 : 464-6.

11. Ishikawa $F$, Takaku $F$, Hayashi $K$, Nagao M, Sugimura T. Activation of rat c-raf during transfection of hepatocellular carcinoma DNA.
Tableau I

GĖNES CELLULAIRES AUTRES QUE CEUX DE LA FAMILLE RAS AYANT DES PROPRIÉTÉS

TRANSFORMANTES SUR LES CELLULES NIH 3 T3

\begin{tabular}{|c|c|c|}
\hline Nom du gène et son origine & Réf. & Observations \\
\hline $\begin{array}{l}\text { 8-lym } \\
\text { Lymphome humain des cellules B. } \\
\text { met } \\
\text { Lignée cellulaire à partir de cellules } \\
\text { d'un ostéosarcome humain activé } \\
\text { par N-méthyl-N'-nitro-N-nitro- } \\
\text { soguanidine. } \\
\text { mcf-2 - mcf-3 } \\
\text { Lignée cellulaire MCF-7 à partir de } \\
\text { cellules d'une tumeur du sein. } \\
\text { c-raf } \\
\text { Tumeur humaine de l'estomac et } \\
\text { hépatocarcinome chimio-induit de } \\
\text { rat } \quad \text { neu } \\
\text { Neuroblastome chimio-induit de rat } \\
\text { dbl } \\
\text { Lymphome humain des cellules B } \\
\text { ret } \\
\text { Lymphome humain des cellules T } \\
\text { Mélanome humain }\end{array}$ & $\begin{array}{l}{[35]} \\
{[36]} \\
{[12]} \\
{[37]}\end{array}$ & $\begin{array}{l}\text { L'activation est consécutive à un } \\
\text { réarrangement du gène au cours de } \\
\text { la transfection [1 1]. } \\
\text { L'activation est consécutive à un } \\
\text { réarrangement du gène au cours de } \\
\text { la transfection }\end{array}$ \\
\hline
\end{tabular}

mise en évidence dans environ $10 \%$ des cas $[9,10]$.

Une autre critique qui peut être faite concerne la méthode d'intégration de l'ADN dans le génome des cellules NIH 3T3, durant laquelle certaines recombinaisons pourraient se faire. C'est effectivement ce qui a été montré, mais uniquement pour d'autres oncogènes que ceux de la famille ras. C'est le cas du gène raf, dont les propriétés transformantes mises en évidence sur les cellules NIH 3T3 viennent d'être attribuées non pas à une mutation, mais à un réarrangement se faisant au cours du transfert de l'ADN tumoral [11]. Un tel réarrangement avait aussi été mis en évidence pour le gène ret [12].

\section{Mutations ponctuelles et augmentation d'expression}

L'étude des gènes ras transformants isolés de tumeurs humaines a permis de montrer que ces oncogènes pouvaient être activés à la suite de mutations ponctuelles (figure 1). Ces dernières ne sont pas localisées au hasard et on a constaté que, pour être actives, elles devaient modifier les propriétés codantes du $12^{\mathrm{e}}$, du $13^{\mathrm{e}}$ ou du $61^{\mathrm{e}}$ codon. Mais il est bien évident que d'autres mutations peuvent exister dans les tumeurs sans donner de gènes transformants décelables par transfection dans les cellules NIH 3T3 ou plus exactement cette technique de transfection ne permet pas de tester des mutations autres que celles en 12 , 13 et 61 , c'est-à-dire des mutations qui ne conferent que des propriétés faiblement transformantes. La mutation de ras n'est cependant pas la seule voie d'activation de l'oncogène : l'augmentation d'expression de gènes ras normaux peut aussi être impliquée dans la cancérogenèse humaine et conduit, lors de la transfection, à un changement de morphologie des cellules NIH 3T3 [13]. Cette augmentation d'expression, qui a été montrée par exemple dans 
certains cancers du sein et plusieurs tumeurs du colon [14-17], peut elle-même être expliquée par deux mécanismes différents. Dans le premier, la région codant pour la protéine peut être placée, à la suite d'un remaniement chromosomique, sous la dépendance du système de régulation d'un autre gène et conduire à une expression importante de l'information génétique du proto-oncogène. Dans le second, le proto-oncogène est répliqué dans les cellules en multiples copies et l'augmentation d'expression est le résultat de cette amplification.

Dans une tumeur gastrique, les deux allèles ras, tous deux activés, le sont par des mécanismes différents : dans l'un, il s'agit d'une mutation ponctuelle, dans l'autre, normal, c'est une amplification de 30 à 50 fois qui est observée [18]. Les auteurs suggèrent que ceci corresponde à deux étapes différentes d'activation. Dans un travail tout récent [19] réalisé à la fois sur une tumeur pancréatique humaine et un ganglion métastasique, une même mutation sur le gène ras ainsi qu'une amplification de 3 à 6 fois du gène activé ont été observées, aussi bien sur la tumeur primaire que sur le ganglion.

\section{Phénomène précoce ou tardif?}

Le fait que les cellules NIH 3T3 soient déjà partiellement transformées et n'auraient besoin que d'une ultime intervention pour devenir complètement malignes a permis d'émettre l'hypothèse selon laquelle l'acquisition des propriétés transformantes par la mutation du gène ras représentait un événement tardif de la tumorigenèse. Certains travaux pouvaient d'ailleurs être interprétés en ce sens $[20,21]$ qui suggéraient que l'acquisition de propriétés de plus en plus malignes se faisait après des passages nombreux de cellules en culture, en relation avec l'activation d'un gène ras.

Cependant, des preuves expérimentales montrant que l'activation du gène ras ne constituait pas toujours un événement tardif dans le développement tumoral ont pu être obtenues grâce à la mise en

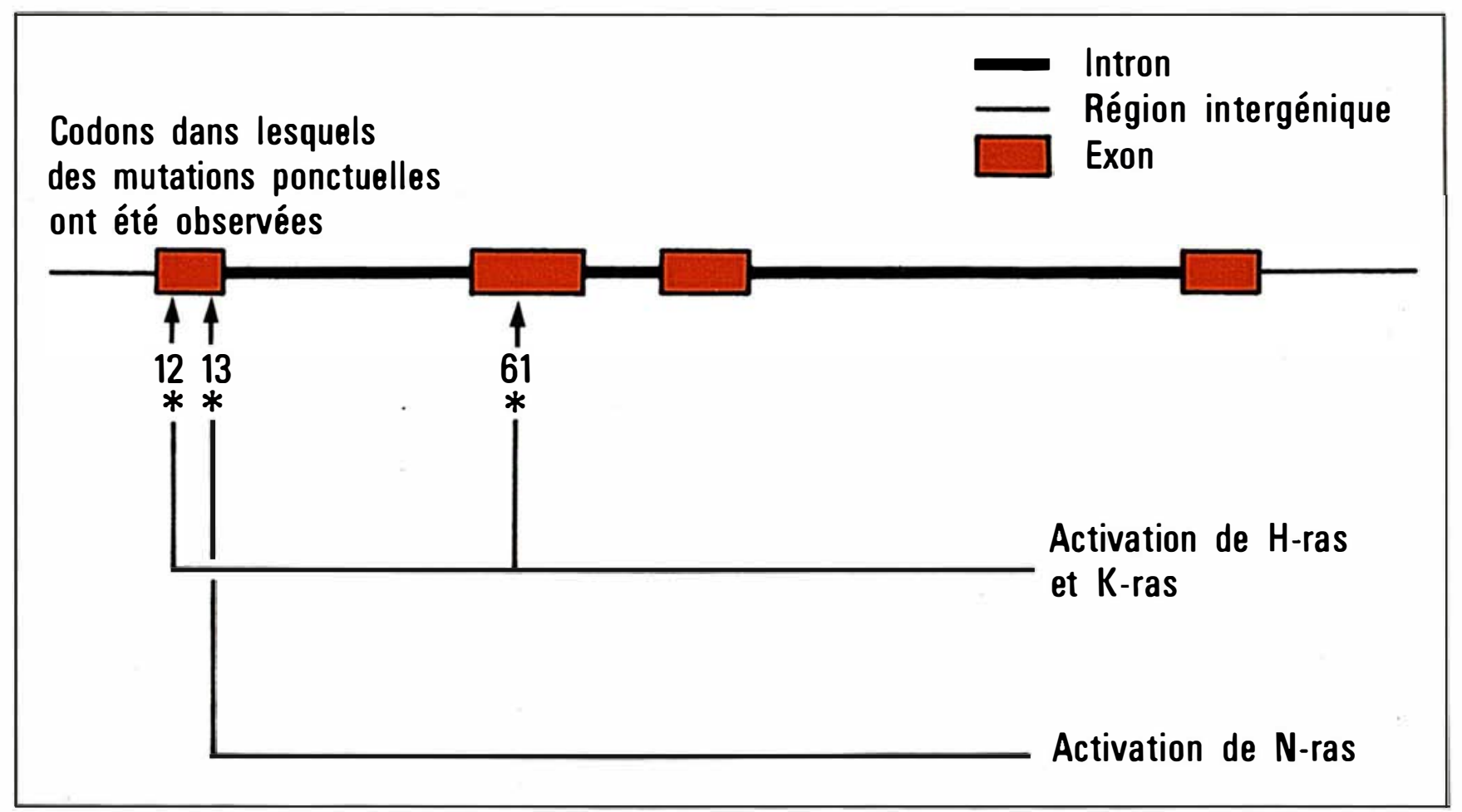

Figure 1. La famille des gènes ras, très conservés au cours de l'évolution, code pour un groupe de protéines, p21, de masse 21000 daltons. Trois membres de cette famille ont été identifiés dans les cellules humaines : H-ras, $K$-ras et $\mathrm{N}$-ras. Les deux premiers, $H$-ras et $K$-ras, ont des équivalents viraux (virus du sarcome murin de Harvey et de Kirstein), le troisième, N-ras, n'en a pas et a été caractérisé dans une lignée établie à partir des cellules d'un neuroblastome humain. L'activation de ces oncogènes est due d̀ des mutations ponctuelles en des sites spécifiques et conduisant à des modifications dans la séquence protéique. A partir de l'ADN extrait de tumeurs humaines, on a pu montrer que, pour $\mathrm{H}$-ras et $\mathrm{K}$-ras, ce sont essentiellement dans les codons codant pour les acides aminés en position 12 et 61 que se trouvent les mutations. Pour le N-ras, la mutation a été observée sur le codon 13. 


\section{RÉFÉRENCES}

12. Takahashi M, Ritz J, Cooper GM. Activation of a nowel human transforming gene, ret, by DNA rearrangement. Cell $1985 ; 42$ : $581-8$

13. Pulciani S, Santos E, Long LK, Sorrentino V, Barbacid M. Ras gene amplification and malignant transformation. Mol Cell Biol 1985 ; 2836-41.

14. Ohuchi N, Thor A, Page DL, Horan Hand P, Halter SA, Schlom J. Expression of the 21,000 molecular weight ras protein in a spectrum of benign and malignant human mammary tissues. Cancer Res 1986; 46 : 2511-9.

15. Spandidos DA, Agnantis N. Human malignant tumors of the breast, as compared to their respective normal tissue, have elevated expression of the Harvey ras oncogene. Anticancer Res 1984 ; 4 : 269-72.

16. Thor A, Horan Hand P, Wunderlich D Caruso A, Muraro R, Schlom J. Monoclonal antibodies define differential ras gene expression in malignant and benign colonic diseases Nature 1984 ; 311 : 562-5.

17. Horan Hand $P$, Thor A, Wunderlich $D$, Muraro R, Caruso A, Schlom J. Monoclonal antibodies of predefined specificity detect activated ras gene expression in human marnmary and colon carcinomas. Proc Natl Acad Sci USA $1984 ; 81: 5227-31$

18. Bos JL, Verlaan-de Vries M, Marshall CJ, Veeneman $\mathrm{GH}$, van Boom $\mathrm{JH}$, van der $\mathrm{Eb} \mathrm{AJ}$. A human gastric carcinoma contains a single mutated and an amplified normal allele of the $\mathrm{Ki}$-ras oncogene. Nucleic Acids Res 1986 ; 14 : 1209-17.

19. Yamada H, Sakamoto H, Taira M, et al. Amplification of both c-Ki-ras with a point mutation and c-myc in a primary pancreatic cancer and its metastatic tumors in lymph nodes. Jpn J Cancer Res, 1986 ; 77 : 370-5.

20. Sukumar S, Pulciani S, Doniger JA, et al. A transforming ras gene in tumorigenic guinea pig cell lines initiated by diverse chemical carcinogenesis. Science 1984 ; 223 : 1197-9.

21. Vousden KH, Marshall CJ. Three different activated ras genes in mouse tumours evidence for oncogene activation of a mouse lymphoma. EMBO J $1984 ; 3$ : 913-7.

22. Kahn A. Hybridation moléculaire : les polymorphismes de taille des fragments de restriction (RFLP, Restriction Fragment Length Polymorphism). médecine/sciences $1986 ; 2$ : 152-3.

23. Quintanilla $M$, Brown $K$, Ramsden $M$, Balmain A. Carcinogen specific mutation and amplification of the $\operatorname{ras}^{\mathrm{H}}$ gene during mouse skin carcinogenesis. Nature 1986 ; 322 : 78-80.

24. Guerrero I, Calzada P, Mayer A, Pellicer A. A molecular approach to leukemogenesis : mouse lymphomas contain an activated c-ras oncogene. Proc Natl Acad Sci USA 1984 ; 81 : 202-5.
25. Sukumar S, Notario V, Martin-Zanca D, Barbacid M. Induction of mammary carcinoma in rats by nitrosomethylurea involves malignant activation of $\mathrm{H}$-ras- 1 locus by single point mutations. Nature $1983 ; 306$ : 658-61.

26. Marshall CJ, Vousden $\mathrm{KH}$, Phillips DH. Activation of c-Ha-ras-l proto-oncogene by in vitro modification with a chemical carcinogen, benzo (a) pyrene diol epoxide. Nature 1984 ; 310 : 586-9.

27. Vousden KH, Bos JL, Marshall CJ, Phillips $\mathrm{DH}$. Mutations activation human $\mathrm{c}-\mathrm{Ha}$ ras-1 proto-oncogene (H RAS 1) induced by chemical carcinogens and depurination. Proc Natl Acad Sci USA 1986 ; 83 : 1222-6.

28. Galiègue-Zouitina S, Bailleul B, Ginot YM, Perly B, Vigny P, Loucheux-Lefebvre $\mathrm{MH} . \mathrm{N}^{2}$ Guanyl and $\mathrm{N}^{6}$ adenyl arylation of chicken erythrocyte DNA by the ultimate carcinogen of 4-nitroquinoline l-oxide. Cancer Res 1986 ; 46 : 1858-63.

29. Rhim JS, Fujita J, Arnstein P, Aaronson SA. Neoplastic conversion of human keratinocytes by adenovirus 12-SV 40 virus and chemical carcinogens. Science $1986 ; 232$ : 385-8.

30. Bargmann CI, Hung MC, Weinberg RA. Multiple independant activations of the neu oncogene by a point mutation altering the transmembrane domain of p 185. Cell 1986 ; 45 : 649-57.

31. Goubin G, Goldman DS, Luce J, Neiman PE, Cooper GM. Molecular cloning and nucleotide sequence of a transforming gene detected by transfection of chicken B-Cell lymphoma DNA. Nature 1983 ; 302 : 114-9.

32. Cooper CS, Park M, Blair DG, et al. Molecular cloning of a new transforming gene from a chemically transformed human cell line. Nature $1984 ; 311$ : 29-33.

33. Fasano D, Birnbaum D, Edlund L, Fogh J, Wigler M. New human transforming genes detected by a tumorigenicity assay. Mol Cell Biol $1984 ; 4$ : 1695-705.

34. Fukui M, Yamamoto T, Kawai S, Maruo K, Toyoshima K. Detection of a raf-related and two other transforming DNA sequences in human tumors maintained in nude mice. Proc Natl Acad Sci USA 1985 ; 82 : 5954-8.

35. Schechter AL, Stern DF, Vaidyanathan L, at al. The neu oncogene : an $\pi b \mathrm{~B}$-related gene encoding a $185,000 \mathrm{M}$ tumor antigen. Nature 1984 ; 312 : 513-6.

36. Eva A, Aaronson SA. Isolation of a new human oncogene from a diffuse B-Cell lymphoma. Nature 1985 ; 273-5.

37. Padua RA, Barras N, Currie GA. A novel transforming gene in a human malignant melanoma cell line. Nature $1984 ; 311: 671-3$

38. Park M, Dean M, Cooper CS et al. Mechanism of met oncogene activation. Cell 1986 ; 45 : 895-904. œuvre de modèles permettant de suivre, sur des animaux, les différentes étapes de la progression tumorale. Le modèle le plus étudié a été celui de la peau de souris sur qui on peut induire des tumeurs par traitement (badigeonnage) avec un cancérogène chimique. Les deux substances qui ont été utilisées, le diméthyl benzanthracène (DMBA) et N-méthyl-N'. nitro-N-nitrosoguanidine (MNNG) provoquent tous deux des tumeurs à la suite d'une unique application. Mais ces deux cancérogènes n'interagissent pas avec l'ADN selon le même mécanisme : l'un, le DMBA, est d'abord métabolisé et provoque surtout des transversions* $\mathrm{A}-\mathrm{T}$, alors que l'autre, le MNNG, est un agent méthylant conduisant à des transitions* G-A. Dans cet essai, on a trouvé pour la majorité des tumeurs induites par le DMBA, un polymorphisme** Xbal sur le ras muté, polymorphisme qu'on ne retrouve pas sur l'oncogène ras des tumeurs induites par le MNNG. Or, si la modification du ras était une conséquence de la progression tumorale, on pourrait s'attendre à ce que sa modification soit la même quel que soit le cancérogène utilisé.

Le résultat obtenu semble donc bien prouver que les mutations sur ras interviennent dès l'étape d'initiation par le cancérogène [23].

\section{Interaction directe avec le cancérogène?}

Un grand nombre d'études réalisées sur des tumeurs humaines [3, $5,9,10,14,15-19]$ ou animales $[21,23-25]$ suggèrent fortement - mais indirectement - que les

\footnotetext{
- Une transition correspond à une mutation dans laquelle une base purique ou pyrimidique est rempla cée par une base de máme nature. Dans une transversion, c'est l'inverse.

* Certaines mutations ponctuelles peuvent intervenir soit dans le site de clivage d'une enzyme de restriction donnée, et le faire ainsi disparaftre, soit créer un nouveau site pour cette enzyme. Il s'ensuit, lors de la digestion enzymatique, une modification de la taille des segments d'ADN, connue sous le nom anglais de RFLP (Restrition Fragment Length Polymor phism). Pour une explication plus détaillée voir [22].
} 
mutations sur le gène ras sont induites par l'action in vivo des cancérogènes chimiques. Une autre approche in vitro a également été réalisée dans le but d'établir une relation causale entre l'action des cancérogènes et l'induction de mutation : c'est celle qui consiste à induire des lésions sur l'ADN du gène ras par action directe avec le cancérogène ultime et, après avoir mis en évidence l'activité du gène ras ainsi modifié sur les cellules NIH 3T3, à caractériser la mutation qui en est responsable et relier sa nature (transversion ou transition) au type de lésion créée par le cancérogène. Le benzo (a) pyrène a été le premier cancérogène chimique à être étudié par cette technique $[26,27]$ : plusieurs mutations localisées soit dans le codon 12 , soit dans le codon 61 ont été identifiées : elles correspondent toutes à des transversions, soit sur la guanine, soit sur l'adénine. Deux des transversions ainsi observées n'avaient pu être mises en évidence dans les systèmes bactériens, qui sont très largement utilisés pour les études du pouvoir mutagène de substances chimiques : autrement dit, les conclusions des études de mutagenèse réalisées sur des systèmes bactériens ne peuvent être généralisées aux systèmes mammiferes, en particulier l'humain.

Il y aurait donc intérêt à étendre ces études à d'autres substances chimiques, ou, plus précisément, à certains autres produits qui seraient choisis d'une part parce que les lésions qu'ils créent sur l'ADN sont parfaitement connues et d'autre part, parce qu'ils peuvent servir de modèle dans la transformation cancéreuse des cellules humaines. Parmi ces cancérogènes, l'oxyde de nitro 4 quinoléine (4NOO) répond à ces deux conditions : 1 'interaction de son cancérogène ultime avec l'ADN a été élucidée [28] et une toute récente publication [29] met en évidence le rôle du $4 \mathrm{NQO}$ dans l'établissement d'une culture de cellules épithéliales tumorales humaines, en faisant ainsi un modèle de cancérogène chimique chez l'homme, dont il faudrait déterminer s'il peut activer un oncogène.

$m / s n^{\circ} 1$ vol. 3 , janvier 87

\section{Mutation ponctuelle dans neu}

Comme cela a été rappelé dans le Tableau I, quelques oncogènes autres que ras sont susceptibles d'induire des foyers dans des cellules NIH 3T3. Leur mécanisme d'activation n'a pas été élucidé sauf, tout récemment, pour l'un d'entre eux, l'oncogène neu [30].

Dans ce cas, c'est aussi l'existence d'une mutation ponctuelle, probablement consécutive à une lésion de l'ADN, qui est responsable de l'activation de l'oncogène : neu a en effet été mis en évidence à partir d'ADN isolé de lignées cellulaires établies à partir de cellules de neuroblastomes chimio-induits chez le rat.

En conclusion, les oncogènes ras activés ont pu être identifiés dans environ $15 \%$ de l'ensemble des tumeurs humaines, quelle que soit leur origine. Leur rôle dans le développement tumoral n'a pas été élucidé et de nombreuses voix se sont élevées pour dire qu'ils n'étaient peut-être activés que dans les dernières étapes de la progression tumorale, comme conséquence du désarroi génétique des cellules cancéreuses. Cependant, les résultats que nous venons de décrire concernant les mutations ponctuelles observées dans un certain nombre de cas attribuent un rôle causal à l'activation de ras dans le développement tumoral. C'est tout au moins ce qui peut être affirmé à présent à la suite des études réalisées sur des modèles animaux, bien que le nombre de mutations mis en évidence soit relativement faible.

Le problème de la détection des mutations et de leur incidence sur l'expression des oncogènes est donc posé. En effet, les mutations qui ont été identifiées l'ont été à la suite d'un avantage de croissance sélectif et les méthodes qui permettent leur étude sont non seulement très peu nombreuses mais aussi parfois très lourdes à mettre en œuvre, comme la transfection sur cellules NIH 3T3. La réalisation de nouveaux essais utilisant des critères biologiques différents permettra de franchir une étape supplémentaire

\section{Summary}

Recent developments in cancer research have shown that the changed activity of certain single genes, termed as cellular oncogenes or proto-oncogenes can lead to neoplastic development. The tumorigenic behavior results from the constitutive switch-on of unmodified cellular oncogenes and/or from modifications in their DNA sequence, due to point mutations or more extensive DNA-rearrangements. In particular the point mutations identified in the ras family of oncogenes are clearly responsible for the acquisition of the transforming potential. This suggests that there may be a direct link between exposure to agents which damage DNA and genetic changes leading to malignancy.

\section{TIRÉS À PART}

M.-H. Loucheux-Lefebvre : Inserm U.124 : Expression des gènes et cancérogenèse chimique, Institut de recherches sur le cancer de Lille, 59045 Lille Cedex. 